\title{
Manno, A. (2020) Toxic Masculinity, Casino Capitalism, and America's Favorite Card Game: The Poker Mindset. Palgrave Macmillan.
}

\section{Review by James V. Catano}

Andrew Manno's Toxic Masculinity, Casino Capitalism, and America's Favorite Card Game: The Poker Mindset performs a number of very important functions. It offers a solid overview of broad aspects of traditional masculinity. It ties these issues to neoliberalism and its underlying encouragement of forms of social behavior. It aligns both of these concepts with a popular analogy linking current capitalist practice with casino play. For those interested in class analysis and masculinist cultural behavior, these are important topics.

In taking up those topics, the book provides an interesting and useful exemplification of both areas by looking at and within the coda of its title: game and gaming behaviors within Poker, aka 'The Poker Mindset' (PM hereafter). Among the concepts comprising the rhetoric of this gendered behavioral set are: Risk and Excitement; Competitiveness; and Courage, Toughness, and Aggressiveness.

After establishing these illustrative components within the first chapter, Toxic Masculinity moves on to an ensuing series of analogies and brief case studies that are of interest to any reader attempting to sort through the threads that make up the intertwined and intersecting threads of contemporary masculine culture, toxic and otherwise. The result is a wide-ranging body of materials including the Alt-Right, media production techniques, the rise of the precariat, populist anger, Martin Shkreli, misogyny in poker, the Men's Rights movement, poker tournaments, Elliott Rodgers and other mass-murderers, masculine pick-up techniques, Incels, and related topics.

Ranging widely about these materials is a large undertaking, of course, and it requires a certain degree of pruning to remove stray parts from each example that might drift outside of the boundaries set up as PM. Perhaps as a result of that need, Toxic Masculinity does have something of a tendency to drift from the part to the whole or, said another way, to substitute the example for the rule. Throughout the book, there is a proclivity to claim a large and all-encompassing role for the book's Poker Mindset.

The Poker Mindset is an ideology, a set of taken-for-granted values that are deeply embedded in American ideas of masculinity and economic success. As with any ideology, these values are perpetuated through cultural training by families, education, religion, politics, and business, just to name a few (p. 47).

But what makes the Poker Mindset-which draws upon and exemplifies the attributes and behaviors in traditional masculinity as noted above - an ideology unto itself and one which 
ostensibly encompasses traditional and toxic masculinity? It is most certainly a useful discussion via analogy, and one which serves to enable general illustrations of masculinism, although such illustrations may fall a bit short of rigorous theoretical description.

A quick example might be found in Chapter 6, 'Fight, Don't Fold': The Poker Mindset and the Rise of Trumpism.' As we are seeing on a daily basis, the ongoing denial by Trump of his loss to Joe Biden certainly enacts (along with splendid examples of his and his bit-players' buffoonery) the bluffing (lying), risk-taking (albeit within the classic sense of entitlement of the certain winner in which there is no risk at all), aggression, winner-take-all mentality that are seen as a part of the Poker Mindset. The question is what such a label provides. PM is certainly a quick and useful thumbnail sketch of Trump. But does it tell us as much as a clinical description of his behavior as 'pathological narcissism and antisocial personality disorder'?

To be sure, not everyone wants to consider the psychological underpinnings of toxic masculinity. But a reader might feel that there is some loss in the tendency to substitute the Poker Mindset of the book's subtitle for the whole behavioral field of masculinity and men's studies. It is a drift that appears in the two-sentence shift of the following, which appears several sentences after the above:

[T]he perfect neoliberal subject embodies traditional masculinity in its expressions of toughness, independence, and aggression. In such a competitive world, success is always possible for those who are seen as deserving by having embraced the values of the Poker Mindset (p. 47).

It may be an unfair overreading to see this slippage as a problem in an overall study attempting to situate where Poker fits into the larger arena of masculinity and its study. All that is required from a reader is to regularly engage in a mental correction of statements so that they are read as follows: 'In particular, this chapter will examine how the hypermasculine ideology [exemplified by] of the Poker Mindset reveals why the Alt-Right is on the rise' (my italics and deletion, p. 48).

But such rewriting might also require noting that the Poker Mindset does not occupy a more commanding role in masculinity than, say 'the Football Mindset' or 'the Boxing Mindset' or 'the Apprentice Mindset.' In fact, such a shift could prove highly useful, allowing for nuanced study of masculinity and the 'Poker Mindset' itself. For example, in considering a hypothetical Football Mindset, it might be possible to talk about how this particular game demonstrates an intensified enactment of 'toughness and aggression' while its nature as a team sport somewhat reduces the emphasis on 'independence.'

Working with such nuances and contradictions is precisely how ideologies work, of course, as they shapeshift and morph large components of masculinity into particular examples, thus ensuring a wide range of applicability within masculine roles while never quite appearing as the perfect enactment of any one form or one analogy. We see such internal ideological conflict in the classic recruitment rhetoric of the US Army, which encourages the possible inductee to 'Be All You Can Be,' even as a key aspect of bootcamp will be the breaking down and replacement of individualism with the overarching identity of the team or squad. 
Such a reading could also serve to mitigate claims that 'It's only by thinking about the ideology of the Poker Mindset - which represents damaging ideas about the roles of men and women, what men are entitled to, and how to achieve success in our neoliberal economy and at what cost - that we can identify ways to combat these ideas on a systemic level' (p. 48). Such overstatement as this risks damaging the book's attempted linkage of attitudes toward poker and toxic masculinity writ large. Surely the Poker Mindset is, as the section's title states, 'A Way' to study masculinity and not, as this sentence seems to imply, the 'only' way?

Refining the discussion along these lines would have a variety of benefits and avoid a process in which the Poker Mindset comes close to being a foundational concept in the production of masculinity. Indeed, the book's introduction rightly notes the situation directly when it defines the Poker Mindset as 'a set of values seen outside the poker table' (p. vii).

The bottom line for the reader is relatively straightforward: if the generalizations to be found in the concept of the Poker Mindset have sufficient descriptive power to elevate them beyond other descriptive approaches, then there is no slippage here and PM is a singularly useful tool for characterizing the wide range of behaviors it notes. If that descriptive power is not there, then the Poker Mindset is not as wide-ranging and elegant as it needs to be to replace other related approaches. Either way, this book contains many useful discussions of its topic materials, and one whose reading will clarify the immense complexity of toxic masculinity and its role in current culture. It does so, moreover, with a wide array of examples, allusions, and references to behaviors and beliefs of particular interest to those concerned with issues of class-inflected identities.

\section{Reviewer Bio}

James V. Catano is producer/director of An Enduring Legacy: Louisiana's Croatian-Americans, author of Ragged Dicks: Masculinity, Steel, and the Rhetoric of the Self-Made Man, and co-editor with Daniel Novak of Masculinity Lessons: Rethinking Men's and Women's Studies. He holds a dual appointment as Professor of English Emeritus, and Professor Screen Arts Emeritus at Louisiana State University. 